\title{
A MULTI-ATTRIBUTE UTILITY MODEL TO SUPPORT SILVICULTURAL DECISION-MAKING IN SECONDARY NORWAY SPRUCE FORESTS
}

\author{
Manfred J. Lexer and Dietmar Jäger \\ Institute of Silviculture \\ University of Agricultural Sciences, Vienna \\ Peter Jordan Strasse 70, A-1190 Vienna - Austria \\ http://www.waldbau.boku.ac.at/ \\ lexer@edv1.boku.ac.at
}

Keywords: Forest transformation, multi-attribute utility model, decision support, analytic hierarchy process, Norway spruce.

\begin{abstract}
Transformation of secondary coniferous forests which had been established at sites naturally supporting broad-leaved species communities to more natural species mixtures which are better adapted to the site currently is a matter of intensive debate in Central European forestry. These secondary conifer forests at low elevations usually are prone to an array of insect and desease organisms. Depending on site conditions multiple rotations of pure Norway spruce may lead to soil compaction, increased soil acidification and decoupled nutrient cycling. The development of sound stand transformation programms for secondary Norway spruce forests usually imposes multiple-criteria decision problems with multiple and partly conflicting objectives. Usually the forest manager is facing a situation where optimal short/midterm utilization of current timber stands has to be compromised with expected long-term benefits from admixed broadleaved species. Due to the involved planning horizons and barely availabe quantitative knowledge on the benefits of stand transformation silvicultural decision making is a challenging task. To avoid schematic and intuitive ad-hoc solutions and to facilitate a rational decision making process a multiple-attribute utility model was developed to meet the requirements of a formal decision analysis. In a case study an additive utility function is employed to aggregate the expected partial utilities from (i) choosing a particular stand treatment option for current stands, and (ii) from the selection of a future species mixture with regard to the partial objectives timber production, sustained site productivity and maintenance of biodiversity. The parameters of the hierarchically structured utility model are estimated with Saaty's eigenvalue method. To avoid the potential problem of rank reversal utility functions were derived for all continously scaled decision criteria. Light conditions within a forest stand and frost occurrence are used as decision constraints to exclude unsuitable combinations of stand treatment options and future species mixtures from the set of eligible alternatives. In a case study the application of the utility model is demonstrated. Sensitivity analysis of the utility model with regard to subjective preferences of the decision maker is pinpointed as a major asset of the implemented approach.
\end{abstract}


